\title{
Desenvolvimento e produtividade da tangerina "Fairchild" sobre quatro porta-enxertos
}

\author{
Development and yield of 'Fairchild' mandarin on four rootstocks
}

\author{
Erick Espinoza-Núñez ${ }^{\mathrm{I}}$ Francisco de Assis Alves Mourão Filho ${ }^{{ }^{*}}$ Eduardo Sanches StuchiI $^{\mathrm{II}}$ \\ Edwin Moisés Marcos Ortega ${ }^{\text {III }}$
}

RESUMO

Neste trabalho foi avaliado o desenvolvimento vegetativo, a produção e a qualidade de frutos da tangerina "Fairchild" [(tangerina "Clementina" (C. clementina Hort. ex. Tan.) x tangelo "Orlando" (C. paradisi Macf. x C. reticulata Blanco)] sobre os porta-enxertos limão "Cravo" (C. limonia Osbeck), citrumelo "Swingle" (P. trifoliata Raf. x C. paradisi Macf.), tangerina "Cleópatra" (C. reshni Hort. ex Tan.) e tangelo "Orlando" (C. reticulata Blanco x C. paradisi Macf.) em Bebedouro, SP, em experimento instalado em 1997. Plantas enxertadas em citrumelo "Swingle" apresentaram menor porte, maior produção acumulada de frutos nas safras 2005 e 2006, maior eficiência de produção $\left(\mathrm{kgm}^{-3}\right)$ e menor índice de alternância de produção. A qualidade dos frutos não foi influenciada em função dos porta-enxertos. O citrumelo "Swingle" foi considerado cultivar porta-enxerto adequado para utilização com tangerina "Fairchild".

Palavras-chave: Citrus, vigor, qualidade.

\section{ABSTRACT}

The vegetative development, fruit yield and quality of 'Fairchild' mandarin [('Clementine' mandarin ( $\boldsymbol{C}$. clementina Hort. ex. Tan.) x 'Orlando' tangelo (C. paradisi Macf. $x$ C. reticulata Blanco)] was evaluated on 'Rangpur' lime (C. limonia Osbeck), 'Swingle' citrumelo (P. trifoliata Raf. x C. paradisi Macf.), 'Cleopatra' mandarin (C. reshni Hort. ex Tan.) and 'Orlando' tangelo (C. reticulata Blanco x C. paradisi Macf.) rootstocks in an experiment installed in Bebedouro, SP, Brazil in 1997. 'Fairchild' trees budded on 'Swingle' citrumelo were smaller, had higher cumulative yield in 2005 and 2006 seasons, higher yield efficiency $\left(\mathrm{kgm}^{-3}\right)$, showed lower alternate bearing index. Fruit quality was not affected by the rootstock. 'Swingle' citrumelo was considered an adequate rootstock for 'Fairchild' mandarin.

Key words: Citrus, vigour, quality.

\section{INTRODUÇÃO}

As plantas cítricas comerciais são formadas pela combinação da copa e do porta-enxerto que se inter-relacionam durante toda sua vida útil, mediante parâmetros, tais como balanço hormonal, relações hídricas e níveis de carboidratos nos tecidos (SYVERTSEN \& LLOYD, 1994). Os porta-enxertos exercem influência no vigor da copa, na produção e no tamanho dos frutos, na qualidade do suco e na tolerância a pragas e doenças, entre diversas outras características (Pompeu Júnior, 2005).

O limão “Cravo” (Citrus limonia Osbeck) é o porta-enxerto mais utilizado na citricultura paulista devido à sua tolerância ao estresse hídrico e ao vírus da tristeza (CTV), ao elevado vigor no viveiro e no campo, à rápida entrada em produção, ao alto rendimento, à maturação precoce dos frutos e à compatibilidade com a maioria das copas cultivadas (POMPEU JÚNIOR, 2005). Por outro lado, árvores vigorosas geralmente produzem frutos grandes, de qualidade interna inadequada, características não- desejáveis em cultivares destinados aos mercados de fruta fresca. Além disso, a utilização de um único porta-enxerto representa elevado risco fitossanitário. Após a detecção da Morte Súbita dos Citros (MSC) nos pomares paulistas, a diversificação dos porta-enxertos passou a ser uma prioridade. Entre as opções para diversificação, citam-se a tangerina "Cleópatra” e o citrumelo “Swingle”, pois, além de não apresentar os sintomas da doença (POMPEU JÚNIOR,

'Departamento de Produção Vegetal, Universidade de São Paulo (USP), Escola Superior de Agricultura “Luiz de Queiroz” (ESALQ), CP 9, 13418-900, Piracicaba, SP, Brasil. E-mail: famourao@esalq.usp.br. * Autor para correspondência.

IIEmbrapa Mandioca e Fruticultura Tropical, Cruz das Almas, BA, Brasil.

IIIDepartamento de Ciências Exatas, USP, ESALQ, Piracicaba, SP, Brasil. 
2005), induzem frutos de melhor qualidade interna em tangerinas (ESPINOZA-NÚÑEZ et al., 2007).

Embora o Brasil seja considerado o maior produtor de citros no mundo, com 20,1 milhões toneladas, pouco mais de 1,3 milhão de toneladas correspondem a tangerinas e híbridos. A China, segundo maior país produtor de citros, é líder na produção de tangerinas, correspondendo a $49 \%$ da produção mundial em 2006 (FAO, 2006). Os principais cultivares e híbridos de tangerinas no Brasil são a tangerina "Ponkan" (C. reticulata Blanco) e o tangor "Murcott” [C. sinensis (L.) Osbeck x C. reticulata Blanco], correspondendo a 80\% do cultivo deste grupo de frutas cítricas no Estado de São Paulo (AMARO \& CASER, 2003).

Os frutos de tangerineiras não se conservam bem nas árvores após completarem a maturação, acarretando em um curto período de colheita (30 a 60 dias), registrando-se preços elevados nos meses de menor oferta. Além disso, considerando-se o aumento significativo das exportações de suco de laranja concentrado, pouca ênfase tem sido dispensada à exploração de mercados locais de fruta fresca no Brasil. Entretanto, com a crescente demanda por produtos naturais e com maior facilidade de processamento, espera-se maior procura por frutos do grupo das tangerinas nos próximos anos (BOTEON \& NEVES, 2005).

A introdução de novos cultivares com características que atendam às expectativas do consumidor, incluindo facilidade de descascamento, tamanho e coloração adequados, poucas sementes, sabor agradável e resistência a doenças, deve ser meta constante por parte do setor produtivo. A tangerina "Fairchild” é cultivar de maturação precoce. Seus frutos são achatados, com casca fina, oleosa, coloração laranja intenso e fortemente aderida, podendo conter elevado número de sementes quando estiver ao redor de cultivares polinizadores (SAUNT, 1990). Ela tem sido reportada como resistente à leprose e clorose variegada dos citros (CVC), o que poderia levar à redução dos custos de produção (LARANJEIRA et al., 2001).

O objetivo deste trabalho foi avaliar o crescimento vegetativo, a produtividade e a qualidade dos frutos da tangerina "Fairchild" sobre quatro portaenxertos.

\section{MATERIAL E MÉTODOS}

O experimento foi realizado na Estação Experimental de Citricultura de Bebedouro (EECB), no município de Bebedouro, SP (latitude 2053’16” S, longitude $48^{\circ} 28^{\prime} 11^{\prime \prime} \mathrm{W}$, altitude $601 \mathrm{~m}$ ), em um solo classificado como Latossolo Vermelho, Distrófico típico, textura média, A moderado, hipoférrico. O clima, segundo a classificação de Köppen, é do tipo Cwa (subtropical com inverno moderado e seco, e verão quente e chuvoso), com temperatura máxima de $28,8^{\circ} \mathrm{C}$, mínima de $18,3^{\circ} \mathrm{C}$ e média de $23,5^{\circ} \mathrm{C}$ e precipitação anual de $1522 \mathrm{~mm}$. As árvores foram plantadas em 30 de outubro de 1997, em densidade de 595 plantas ha-1 (7,0m $\mathrm{x} 2,4 \mathrm{~m})$. As plantas foram cultivadas de acordo com as recomendações técnicas do cultivo, entretanto, não foram realizadas irrigações, podas e desbaste de frutos. O pomar experimental está rodeado por vários outros experimentos com plantas cítricas.

A tangerina "Fairchild", um híbrido entre tangerina "Clementina” (C. clementina Hort. ex. Tan.) e tangelo “Orlando" (C. paradisi Macf. x C. reticulata Blanco), foi enxertada sobre os porta-enxertos limão "Cravo" (Citrus limonia Osbeck), citrumelo "Swingle" (P. trifoliata Raf. x C. paradisi Macf.), tangelo “Orlando” (C. reticulata Blanco x C. paradisi Macf.) e tangerina “Cleópatra” (Citrus reshni Hort. ex Tan.).

Na data da colheita, realizada em julho de cada ano, registrou-se a produção total de frutos por planta, calculando-se a produção acumulada para todos os anos e separadamente para as safras 2005 e 2006. A análise de qualidade foi realizada no Laboratório de Qualidade da EECB, cujas amostras foram compostas por cinco frutos por planta, determinando-se a massa média dos frutos em uma balança com sensibilidade de cinco g. O suco foi extraído por extratora OIC modelo OTTO 1800, calculando-se a porcentagem do suco pela relação massa do suco / massa do fruto e multiplicada por 100 .

O teor de sólidos solúveis totais (SST) foi determinado por leitura direta no refratômetro. A acidez total (AT) foi obtida por titulação de $25 \mathrm{ml}$ de suco com hidróxido de sódio 0,3125 N. O Ratio foi calculado pela relação entre SST e AT. O volume da copa (V) foi calculado pela coleta das variáveis altura da planta $(\mathrm{H})$, diâmetro da copa no sentido da linha (Dl) e no sentido 
perpendicular à rua (Dr) em agosto de 2006, e aplicação da fórmula: $\mathrm{V}=(\pi / 6) \times \mathrm{H} \times \mathrm{Dl} \times \mathrm{Dr}$ (TURREL, 1946). $\mathrm{O}$ diâmetro do tronco da copa foi medido $10 \mathrm{~cm}$ acima do ponto de enxertia em agosto de 2006. A eficiência da produção foi estimada dividindo-se a produção de frutos pelo volume da copa induzida por cada portaenxerto em 2006.

Devido à previsível irregularidade da produção nos primeiros anos em plantas cítricas, incluindo tangerinas, o índice de alternância da produção (IAP) foi calculado entre os anos 2003 e 2006 pela fórmula: IAP $=1 / n-1 \times\left\{\left|\left(a_{2}-a_{1}\right)\right| /\left(a_{2}+a_{1}\right)+\left|\left(a_{3}-a_{2}\right)\right| /\right.$ $\left.\left(\mathrm{a}_{3}+\mathrm{a}_{2}\right)+\ldots+\left|\left(\mathrm{a}_{(\mathrm{n})}-\mathrm{a}_{(\mathrm{n}-1)}\right)\right| /\left(\mathrm{a}_{(\mathrm{n})}+\mathrm{a}_{(\mathrm{n}-1)}\right)\right\}$ (STENZEL et al., 2003), em que $n=$ número de anos e $a_{1}, a_{2}, \ldots, a_{(n-1)}, a_{(n)}=$ produção dos anos correspondentes.

O delineamento estatístico foi inteiramente aleatorizado, com quatro tratamentos (porta-enxertos), com cinco repetições e uma planta por parcela, totalizando 20 plantas. As plantas foram distribuídas em uma linha, sem bordaduras. A produção anual, a produção acumulada total e a acumulada em 2005 e 2006, o diâmetro do tronco, o volume de copa, a eficiência de produção, o índice de alternância da produção e a qualidade dos frutos foram submetidas à análise de variância e as médias foram comparadas pelo teste de Tukey a 5\% de probabilidade de erro.

\section{RESULTADOS E DISCUSSÃO}

Não foram observadas diferenças na produção de frutos em função dos porta-enxertos na maioria das safras estudadas. Em 2000, as plantas sobre limão “Cravo” apresentaram maior produção de frutos, correspondendo a mais que o dobro daquelas sobre o citrumelo "Swingle” e tangelo “Orlando" (Tabela 1). Em 2003, as plantas enxertadas em tangelo “Orlando”, tangerina “Cleópatra” e citrumelo "Swingle” produziram maior quantidade de frutos quando comparadas àquelas sobre limão “Cravo”. Na produção acumulada total dos frutos, não ocorreram diferenças em função dos porta-enxertos. Por outro lado, as plantas sobre o citrumelo "Swingle" e limão "Cravo" obtiveram a maior produção acumulada nas safras 2005 e 2006, sendo que aquelas sobre citrumelo "Swingle" foram $58 \%$ e $76 \%$ mais produtivas do que aquelas sobre tangelo “Orlando” e tangerina “Cleópatra”, respectivamente. Este fato está de acordo com investigações realizadas em Chipre, em que o citrumelo "Swingle” foi similar ao limão "Cravo” na produção acumulada de 11 anos de tangerina "Nova" (GEORGIOU, 2000).

Pesquisas realizadas no Rio Grande do Sul indicaram que plantas de tangerina "Montenegrina" sobre citrumelo "Swingle" produziram mais frutos quando comparada com aquelas sobre citrange “Troyer” e Trifoliata (SHÄFER et al., 2001). Avaliações da tangerina "Fairchild" no Arizona, Estados Unidos, indicaram menor produção de frutos sobre tangerina "Batangas" quando comparadas com aquelas sobre limões e citranges (FALLAHI \& RODNEY, 1992).

As plantas sobre citrumelo "Swingle" apresentaram produção mais constante que aquelas

Tabela 1 - Produção anual e acumulada de frutos, índice de alternância de produção (IAP), diâmetro de tronco, volume de copa e eficiência de produção de árvores da tangerina "Fairchild” sobre quatro porta-enxertos, Bebedouro, SP (2000-2006).

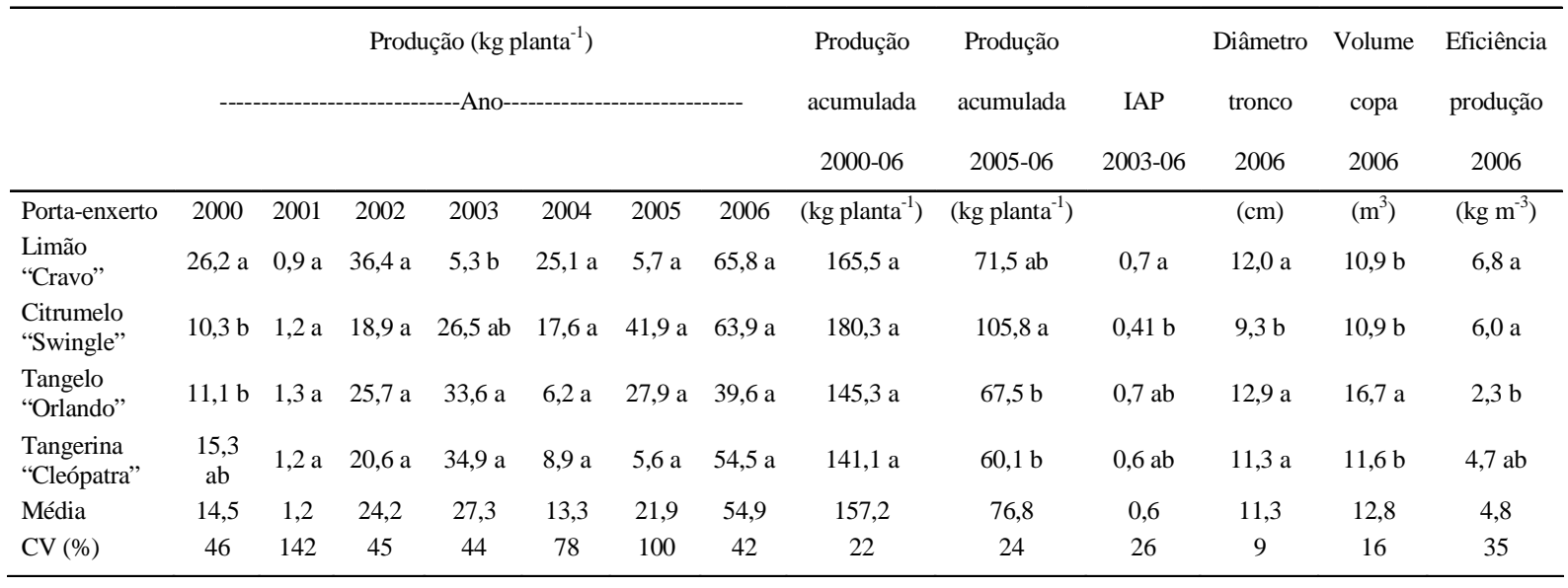

Médias não seguidas da mesma letra na coluna diferem pelo teste de Tukey em nível de 5\% de probabilidade de erro. 
sobre limão "Cravo", de acordo com o índice de alternância de produção (Tabela 1), concordando com pesquisas anteriores em tangerina "Ponkan”, no Paraná, em que a alternância da produção foi menor sobre citrange C13 e Trifoliata (STENZEL et al., 2003). Por outro lado, vários trabalhos não reportaram efeitos dos porta-enxertos nesta característica (GEORGIOU, 2000; SMITH et al., 2004; MOURÃO FILHO et al., 2007). Nos anos “on” (alta produção), métodos para controlar a carga de frutos são recomendados para amenizar a alternância, tais como raleio de frutos, anelamento de ramos, aplicações exógenas de reguladores vegetais e antecipação da colheita. Nos anos “off” (baixa produção), a floração pode ser incrementada com aplicações foliares de uréia (EL-OTMANI et al., 2004).

As plantas mais vigorosas foram aquelas sobre tangelo "Orlando” com base no volume de copa. Por outro lado, as plantas sobre citrumelo "Swingle" apresentaram os menores valores de diâmetro de tronco em relação àquelas sobre os demais porta-enxertos (Tabela 1). Árvores das tangerinas "Page” e "Robinson” sobre os porta-enxertos tangelo "Orlando" e tangerina “Cleópatra” também apresentaram maior tamanho (HEARN \& HUTCHISON, 1977). Pesquisas no Brasil têm indicado que a utilização de tangerinas como porta-enxertos para tangerinas leva à formação de plantas de maior porte (STENZEL et al., 2003; ESPINOZA-NÚÑEZ et al., 2007).

A eficiência de produção foi menor em plantas sobre tangelo "Orlando", resultante do maior volume de copa. O tangelo “Minneola” utilizado como copa apresentou alta eficiência de produção sobre limão "Cravo” e citrumelo "Swingle” na Califórnia (ROOSE, 1996). As árvores com maior copa normalmente apresentam maior capacidade produtiva se as condições de luminosidade são as adequadas (WHEATON et al., 1978), fato não confirmado para o tangelo “Orlando” (Tabela 1).

Embora trabalhos anteriores tenham revelado a influência do citrumelo "Swingle" na qualidade de frutos de tangerinas (ESPINOZA-NÚÑEZ et al., 2007), neste experimento não se verificou influência de nenhum porta-enxerto nas características da qualidade do suco dos frutos nas duas safras (2005/ 2006) (Tabela 2). O efeito dos porta-enxertos sobre a qualidade dos frutos é reduzido quando as plantas se desenvolvem em solos bem manejados e de textura média, quando comparados aos solos arenosos (CASTLE, 1995). O solo Latossolo Vermelho de textura média na EECB oferece condições físicas adequadas para o desenvolvimento radicular profundo das plantas, assim como grande capacidade de armazenamento de água, fato que poderia explicar os resultados deste experimento.

\section{CONCLUSÕES}

O citrumelo "Swingle” é cultivar portaenxerto adequado para utilização com tangerina "Fairchild".

Tabela 2 - Massa do fruto, porcentagem de suco, sólidos solúveis totais (SST), acidez total (AT) e ratio de frutos da tangerina "Fairchild" sobre quatro porta-enxertos Bebedouro, SP (2005 - 2006).

\begin{tabular}{|c|c|c|c|c|c|c|c|c|c|c|}
\hline \multirow[t]{2}{*}{ Porta-enxerto } & \multicolumn{2}{|c|}{ Massa do fruto (g) } & \multicolumn{2}{|c|}{ Porcentagem de suco (\%) } & \multicolumn{2}{|c|}{ SST (\%) } & \multicolumn{2}{|c|}{ AT (\%) } & \multicolumn{2}{|c|}{ Ratio } \\
\hline & 2005 & 2006 & 2005 & 2006 & 2005 & 2006 & 2005 & 2006 & 2005 & 2006 \\
\hline Limão "Cravo" & $113,5 \mathrm{a}$ & 147,7 a & 49,0 a & 52,0 a & $14,8 \mathrm{a}$ & 13,8 a & $1,2 \mathrm{a}$ & $0,9 \mathrm{a}$ & 12,6 a & $14,2 \mathrm{a}$ \\
\hline $\begin{array}{l}\text { Citrumelo } \\
\text { "Swingle" }\end{array}$ & $141,2 \mathrm{a}$ & 151,3 a & $48,8 \mathrm{a}$ & 51,7 a & $14,2 \mathrm{a}$ & $13,8 \mathrm{a}$ & $1,2 \mathrm{a}$ & 0,9 a & $12,2 \mathrm{a}$ & $14,8 \mathrm{a}$ \\
\hline Tangelo "Orlando" & 144,9 a & 150,8 a & 49,7 a & 52,7 a & $14,2 \mathrm{a}$ & 13,4 a & $1,2 \mathrm{a}$ & 0,9 a & 11,6 a & $13,8 \mathrm{a}$ \\
\hline $\begin{array}{l}\text { Tangerina } \\
\text { “Cleópatra” }\end{array}$ & 129,7 a & $147,5 \mathrm{a}$ & 49,4 a & 54,1 a & 14,3 a & 13,6 a & 1,3 a & 0,9 a & $10,7 \mathrm{a}$ & $14,3 \mathrm{a}$ \\
\hline Média & 135,6 & 149,3 & 49,3 & 52,6 & 14,3 & 13,6 & 1,2 & 0,9 & 11,6 & 14,3 \\
\hline CV (\%) & 12 & 4 & 2 & 2 & 4 & 3 & 10 & 7 & 12 & 8 \\
\hline
\end{tabular}

Médias não seguidas da mesma letra na coluna diferem pelo teste de Tukey em nível de 5\% de probabilidade de erro.

Ciência Rural, v.38, n.6, set, 2008. 


\section{REFERÊNCIAS}

AMARO, A.A.; CASER, D.V. Diversidade do mercado de tangerinas. Informações Econômicas, São Paulo, v.33, n.12, p.51-67, 2003.

BOTEON, M.; NEVES, E.M. Citricultura brasileira: aspectos econômicos. In: MATTOS JUNIOR, D. et al. (Org.). Citros. Campinas: Instituto Agronômico/FUNDAG, 2005. p.19-36.

CASTLE, W.S. Rootstock as a fruit quality factor in citrus and deciduous tree crops. New Zealand Journal of Crop and Horticultural Science, Wellington, v.23, n.3, p.383-394, 1995.

EL-OTMANI, M. et al. Improved use of foliar urea on Clementine mandarin to manipulate cropping in a sustainable production system. Acta Horticulturae, The Hague, v.632, p.167-175, 2004.

ESPINOZA-NÚÑEZ, E.N. et al. Desenvolvimento vegetativo, produção e qualidade de frutos da tangerina "Fremont" sobre quatro porta-enxertos. Revista Brasileira de Fruticultura, Jaboticabal, v.29, n.2, p.308-312, 2007.

FALLAHI, E.; RODNEY, D.R. Tree size, yield, fruit quality, and leaf mineral nutrient concentration of Fairchild mandarin on six rootstocks. Journal of the American Society for Horticultural Science, Geneva, v.117, n.1, p.28-31, 1992.

FAO. FAOSTAT: Statistical database. Capturado em 17 ago. 2006. Online. Disponível na Internet http://faostat.fao.org/.

GEORGIOU, A. Performance of 'Nova' mandarin on eleven rootstocks in Cyprus. Scientia Horticulturae, Amsterdam, n.84, p.115-126, 2000.

HEARN, C.J.; HUTCHINSON, D.J. The performance of Robinson and Page citrus hybrids on 10 rootstocks. Proceedings of the Florida State Horticultural Society, Tallahasse, n.90, p.44-47, 1977.

LARANJEIRA, F.F. et al. Tangelos: híbridos altamente resistentes à leprose e à clorose variegada dos citros.
Fitopatologia Brasileira, Brasília, v.26, Suplemento, p.278, 2001.

MOURÃO FILHO, F.A.A. et al. Plant growth, yield, and fruit quality of 'Fallglo' and 'Sunburst' mandarins on four rootstocks. Scientia Horticulturae, Amsterdam, n.114, p.45-49, 2007.

POMPEU JÚNIOR, J. Porta-enxertos. In: MATTOS JUNIOR, D. et al. (Org.). Citros. Campinas: Instituto Agronômico/ FUNDAG, 2005. p.63-104

ROOSE, M.L. Performance of 4 citrus scions on 21 rootstocks in California. In: INTERNATIONAL CITRUS CONGRESS, 1996, Sun City. Proceeding... Sun City: International Society of Citriculture, 1996. p.141-144.

SAUNT, J. Citrus varieties of the world. Norwich: Sinclair International, 1990. 128p.

SCHÄFER, G. et al. Produção e desenvolvimento da tangerineira "Montenegrina” propagada por enxertia e estaquia, no Rio Grande do Sul. Revista Brasileira de Fruticultura, Jaboticabal, v.23, n.3, p.668-667, 2001.

SMITH, M.W. et al. Long-term performance of "Ellendale" mandarin on seven commercial rootstocks in sub-tropical Australia. Scientia Horticulturae, Amsterdam, v.102, p.7589, 2004.

SYVERTSEN, J.P.; LLOYD, J.L. Citrus. In: SHAFFER, B.; ANDERSEN, P.C. Handbook of environmental physiology of fruit crops. Boca Raton, Fla: CRC, 1994. p.65-99.

STENZEL, N.M.C. et al. Performance of "Ponkan" mandarin on seven rootstocks in Southern Brazil. Hortscience, St Joseph, v.38, n.2, p.176-178, 2003.

TURRELL, F.M. Tables of surfaces and volumes of spheres and of prolate and oblate spheroids and spheroidal coefficients. Berkeley: University of California, 1946. 153p.

WHEATON, T.A. et al. Higher density for Florida citrus concepts. P roceedings of the Florida State Horticultural Society, Tallahasse, n.91, p.17-33, 1978. 